\title{
IMAGINARIOS AMBIENTALES: VOCES EMERGENTES DESDE LA VIDA COTIDIANA
}

\author{
Luz Elena Ospina O. ${ }^{\mathrm{i}}$ \\ Napoleón Murcia P ii \\ ${ }^{\text {i }}$ Profesional en Desarrollo Familiar. Tecnóloga en Sistemas Informáticos. Aspirante a \\ Magíster en Educación con énfasis en Multimedia Educativo. E-mail: \\ apo3leo@gmail.com \\ ii Magister en educación, $\mathrm{PhD}$. en ciencias sociales niñez y juventud. Profesor \\ Universidad de Caldas. Director departamento de estudios educativos. Coordinador \\ grupo educación, motricidad y mundos simbólicos. E mail: napo2308@gmail.com
}

Manizales, 2008-04-20 (Rev. 2008-06-18)

\section{RESUMEN}

El presente escrito está fundamentado en la investigación titulada "Vida ambiental universitaria. Una construcción desde los imaginarios cotidianos", inscrito en la Vicerrectoría de Investigaciones y Postgrados de la Universidad de Caldas en octubre del año 2006, el cual pretende comprender los imaginarios que sobre el ambiente se entretejen en el bullicio del cotidiano universitario.

Un primer acercamiento a los imaginarios ambientales en la Universidad de Caldas fue realizado desde fuentes documentales institucionales como: el Proyecto Educativo Institucional, la Revista Luna Azul y Catálogos de Investigación; este permitió navegar por los sentidos generales que dibujan los imaginarios irradiados en el perímetro instituciona (1). Son relevantes en esta revisión como imaginarios instituidos (2), entre otros: la protección ambiental, la identificación de los responsables de problemas ambientales, la caracterización de la naturaleza (animada e inanimada) y la educación ambiental. Como imaginarios instituyentes (3) sobresalen: formas de educación alterna (es decir diferente a los estilos tradicionales o previstos por la ley), relación interdependiente del ser humano y la naturaleza, la imaginación como potencia de cambio, y transformación y construcción del futuro.

Un segundo acercamiento entraña el flujo de imaginarios ambientales desde la cotidianidad universitaria. Los resultados muestran una fuerte configuración de imaginarios instituidos a partir de la concepción del ambiente como naturaleza (flora y fauna generalmente), una segunda configuración de imaginarios instituyentes fue dibujada desde aspectos sociales orientados hacia la cultura; y una tercera configuración desde imaginarios radicales $(4)$, orientada desde la noción de magma, entorno (todo lo que nos rodea) y tecnología.

\section{PALABRAS CLAVE}

Ambiente, imaginarios, cotidianidad, universidad, vida ambiental, imaginarios ambientales, cotidianidad universitaria, magma, naturaleza, cultura, tecnología, entorno. 


\title{
ENVIRONMENTAL IMAGINARIES: EMERGENT VOICES FROM DAILY LIFE
}

\begin{abstract}
The present writing is based on the ongoing study entitled "university environmental Life. A construction based on daily life imaginaries", registered in the Vice-rectory or Research and Postgrades of the Universidad de Caldas in October 2006, which attempts to understand imaginaries constructed regarding the environment within the clatter of daily college life. A first approach to environmental imaginaries at the Universidad de Caldas was carried out using institutional documentary sources such as the Institutional Educative Project, Luna Azul Journal and research catalogues, permitting to sail through the general senses drawn by the imaginaries irradiated within the institutional perimeter. In this revision, instituted imaginaries include environmental protection, the identification of the people responsible for environmental problems, the characterization of nature (animated and unanimated) and environmental education. Instituting imaginaries include: alternate education forms, which is to say, different from traditional styles, interdependent relationship between humans and nature, imagination as a change, transformation and construction potent for the future. A second approach involves the flow of environmental imaginaries from university daily life. The results show a strong configuration of instituted imaginaries from the conception of seeing the environment as nature (generally flora and fauna), a second configuration of instituting imaginaries was drawn from social aspects oriented towards culture; and a third configuration based on radical imaginaries, connected to the notion of magma, surroundings (everything what surrounds us) and technology.
\end{abstract}

\section{KEY WORDS}

Environment, imaginaries, daily life, university, environmental life, environmental imaginaries, university daily life, magma, nature, culture, technology, surroundings.

\section{ESTRATEGIA METODOLÓGICA}

El enfoque utilizado fue el de complementariedad etnográfica, desde el cual se reconoce la complejidad de las realidades sociales y la necesidad del apoyo en diferentes teorías y métodos de recolección y procesamiento, siempre que ayuden a la comprensión profunda de la realidad social estudiada. En el diseño e interpretación no se trata de yuxtaponer los datos cuantitativos y cualitativos, sino de articularlos en una interpretación que busque profundidad y amplitud. El dato funge, en esta perspectiva, como un instrumento de información y, al articularse con la información sustantiva, ayuda al propósito de comprensión de la realidad (Murcia y Jaramillo 2007).

El diseño transitó por dos momentos: en el primero, se acudió al análisis documental utilizando para el procesamiento fichas de contenido y cuadros de categorización simple, axial y selectiva, en el segundo, se realizaron entrevistas y encuestas. Los dos 
acercamientos fueron enriquecidos con elementos de análisis semántico, análisis de sentidos y análisis del discurso escrito y oral. Lo cual permitió la construcción de categorías imaginarias ambientales que paulatinamente permitieron configurar un prototipo de imaginario instituido, instituyente o radical.

Se realizaron 8 entrevistas en profundidad a similar número de profesores, 12 a estudiantes, 6 a directivos y 6 a otros funcionarios (secretarias, vigilantes y de oficios varios). Para la selección de los entrevistados se tuvo en cuenta criterios como: antigüedad en la institución, área de conocimiento, estamento y sede. Los encuestados fueron elegidos bajo los requerimientos de un muestreo probabilístico aleatorio estratificado, en el cuál el universo es constituido por subgrupos de la población, en razón a lo cual el resultado muestral se definió en el siguiente cuadro:

Cuadro $\mathbf{N}^{\mathbf{0}}$ 1. Cuadro que determina el tamaño muestral y el universo del cuál fu extraído

\begin{tabular}{|l|c|}
\hline Población/Universo $\underline{(\mathbf{5})}$ & Muestra $\underline{\mathbf{( 6 )}}$ \\
\hline 10.723: Estudiantes de pregrado (presenciales y a distancia) & 568 \\
\hline 615: Profesores (planta: 410; ocasional: 205$)$ & 304 \\
\hline 177: Otros funcionarios (trabajadores: 87 ; secretarias: 90$)$ & 137 \\
\hline TOTALES & 1009 \\
\hline
\end{tabular}

Los datos fueron calculados bajo los siguientes parámetros:

- Nivel de confianza: $95 \%$

- Variabilidad negativa y positiva: 0.5 cada una

- Porcentaje de error: $4 \%$

La fórmula aplicada es la recomendada por la teoría de muestreo para investigaciones sociales:

$$
n=\frac{Z^{2} p q N}{N E^{2}+Z^{2} p q}
$$

Donde:

$n$ es el tamaño de la muestra;

$Z$ es el nivel de confianza;

$p$ es la variabilidad positiva;

$q$ es la variabilidad negativa;

$N$ es el tamaño de la población;

$E$ es la precisión o el error. 


\section{ACERCAMIENTOS TEÓRICOS}

\section{IMAGINARIOS/UNIVERSIDAD}

Antes de iniciar con los hallazgos del segundo acercamiento a los imaginarios ambientales en la Universidad de Caldas, es prudente hacer una breve distinción de la perspectiva bajo la cual se fundamentan los imaginarios que aquí se tratan:

Imagen, representación social, simbólico; son conceptos que se deben llenar de significado para poder establecer alguna claridad sobre lo que se considera como imaginario social.

Los imaginarios corresponden a esa categoría de la conciencia que transita entre estos conceptos y que los hace posibles como manifestación de la compleja imbricación entre lo deseado y lo factible. Es entones una construcción más que subjetiva, intersubjetiva e intrasubjetiva que va mas allá de la representación de las características denotativas del objeto o fenómeno, y que implica la apropiación de estas desde la historia de vida de quien le da el sentido. Por eso, el imaginario, es más que la subjetivación de la imagen, puesto que ésta se relaciona con la representación y aquella con el sentido otorgado a toda una vida de formas, sentimientos, percepciones, motivos para las acciones e interacciones, necesidades y deseos (Murcia, 2006, p. 19).

Los seres humanos desde las múltiples relaciones logradas en el mundo de la vida, vamos representando la realidad en imágenes que al ser cargadas de sentido conforman el mundo de lo imaginario "pregnancia simbólica" diría E. Cassirer (1971), pero ese proceso de pregnancia simbólica no se produce linealmente, sino que es un entramado complejo de relaciones de significado, significante, deseo y posibilidad.

Pese a que el imaginario se expresa en lo simbólico, en el mundo de lo imaginario, lo simbólico es constituido y constituyente, pues se constituye a partir de la carga de sentido otorgada a la imagen, y constituye en la medida que es también desde una cierta consideración simbólica que se forman los imaginarios "los sentidos simbólicos están abiertos a nuevas disposiciones de significación”. Sin embargo, y por encima de las atribuciones a veces unidireccionales, que le han dado a lo simbólico como representación directa de lo imaginario, o a lo imaginario como consecuencia de lo simbólico, estos se resignifican entre sí permanentemente, en el mundo de la vida de los sujetos.

En síntesis, los imaginarios se refieren a esa carga intangible de sentido que las personas le damos al mundo y a nosotros mismos y desde las cuales organizamos nuestras vidas. Es lo que asumimos como realidad.

Las aproximaciones al concepto son variadas, aunque siempre toman en común: la relación cercana con lo simbólico en tanto son su forma de expresarse, la representación que hace las veces de mediador que permite presentar lo simbólico, la intangibilidad de lo imaginario en tanto sustantividad y el imaginario social, como única opción de inteligibilidady plausibilidad social. 
Mientras, para Durand (1968, p. 6) los imaginarios son un conjunto de imágenes que constituyen el capital pensado del ser humano y el cual se ubica como elemento fundamental en el establecimiento de las relaciones con otros individuos y con el espacio. O sea son las estructuras estables que se han ido acumulando en la sociedad el cual logra su máxima expresión cuando se construye en ausencia del objeto "la imaginación simbólica". No se puede representar "en cuanto tal, una palabra exacta o una descripción única, y lo que se presenta es más que una cosa, un sentido o muchos que pueden abarcar la expresión simbólica".

Para Shotter, implica esas entidades políticas que aun no existen del todo- pero que tampoco son del todo ficticias:

“(...) de acuerdo con las cuales organizamos retóricamente nuestras relaciones sociales(...) en un principio, la existencia de esas entidades políticas reside en su mera subsistencia en lo que la gente dice de ellas, pero-en la medida en que nuevas formas de hablar tienden a "construir" nuevas formas de relación social- empiezan a asumir una existencia mas "real" (normalmente intransigente) cuanto más se habia cuanto más se habla "de" ellas, y dan origen a nuevas instituciones y estructuras sociales". (Shotter 1993, p. 29).

Son entidades políticas justamente porque ayudan a constituir, porque no son entidades definidas o ya constituidas sino que son generativas de una forma de organización del mundo. Son constructoras de esas identidades sociales desde las cuales las personas organizan sus vidas y valoran la organización de la vida de los otros. Es aquello que cruza la definición de los valores y normas que permiten inteligibilidad y plausibilidad social; son imaginarios en el sentido de hallarse solamente en los procesos que se producen entre las personas.

Para Castoriadis (1983) el imaginario es la capacidad de la sociedad y de la psique-soma de generar, crear, imaginar su propia red simbólica. Es entonces esa sustantividad histórico/social que pregna las funciones sociales y que se expresa siempre desde los simbólicos que la sociedad construye:

"...hablamos de imaginario cuando queremos hablar de algo "inventado"- ya se trate de un invento absoluto (una historia imaginada de cabo a rabo) o de un deslizamiento, de un desplazamiento de sentido, en el que unos símbolos ya disponibles están investidos con otras significaciones que las suyas "normales" o canónicas... En los dos casos, se da por supuesto que lo imaginario se separa de lo real. (Castoriadis 1983, p. 219)

Por eso lo imaginario siempre utiliza lo simbólico para expresarse y viceversa, lo simbólico presupone también la capacidad imaginaria de decir algo como si fuera tal. El autor propone tres tipos de imaginarios, los imaginaros instituidos, los imaginarios instituyentes y los imaginarios radicales, anteriormente descritos.

Analizando los imaginarios ambientales universitarios, desde los imaginarios planteados por Castoriadis, diríamos que: los imaginarios ambientales/instituidos son aquellos que inculcan la creencia en que el orden en el cual vivimos es "natural" o nos ha sido dado por dioses, antepasados idealizados, leyes económicas, modos de producción, entre otros, y son incuestionables; además se distinguen por dos aspectos: uno, por ser 
estructuras en las cuales se determina unidireccionalidad de comportamientos e intenciones, por tanto hay una norma que regula y hay un conjunto notable de seguidores que no cuestionan la norma; y, dos, la temporalidad, los imaginarios instituidos difícilmente hacen rupturas, esguinces que le permita virar hacia otros horizontes, por eso se hace permanente en el tiempo.

Los imaginarios ambientales/instituyentes son aquellos en los que la exploración, la imaginación dan origen a la creación, creación que identifica el o los modos de ambiente/Universidad, modos que emergen desde las diferentes significaciones (sentidos, significados, actuaciones, pensares, sentires, etc.) que los integrantes de la Universidad otorgan o vivencian frente al ambiente y dan el toque de originalidad a la institución.

Ambos imaginarios, visualizan los profundos, invisibilizados o más bien implícitos movimientos que avivan, y dibujan la mirada del ambiente en la Universidad de Caldas. Estos imaginarios son los que a continuación se pretenden compartir. Es de aclarar que tales imaginarios emergen desde el mundo de la cotidianidad universitaria, desde el acontecer universitario, desde "la forma real en que se viven los valores, creencias, aspiraciones y necesidades" (Heller, 1972) frente al ambiente en la Universidad de Caldas.

\section{HALLAZGOS}

\section{IMAGINARIOS AMBIENTALES QUE IRRADIAN LA UNIVERSIDAD DE CALDAS}

Este primer encuentro con los imaginarios ambientales de la Universidad de Caldas está ampliamente tratado en el artículo que lleva por título el mismo nombre del apartado, publicado en la revista electrónica institucional de la Universidad de Caldas, Luna Azul. No obstante, se esbozarán las ideas centrales del escrito a fin de dar el panorama, o más bien el techo bajo el cual hacen ebullición los imaginarios ambientales de la comunidad universitaria.

Hacia la década de los 70` se hace visible un flujo de pensamientos alrededor de lo denominado como crisis ambiental, dichos pensamientos reflejan imaginarios que paulatinamente se han filtrado en las leyes e instituciones de la sociedad hasta llegar a la Universidad de Caldas, estos imaginarios son:

1. Volver al primitivismo (7) : Corriente asociada normalmente con los ecologistas radicales, cuyos objetivos se centran en conseguir un estado asilvestrado a través de la resalvajización; aboga por volver a una vida no civilizada a través de la desindustrialización, la abolición de la división del trabajo y el abandono de la tecnología.

2. Construir un ideal de ecodesarrollo: Los pensamientos asociados con el ecodesarrollo pretenden una articulación armónica entre el crecimiento económico y la naturaleza.

3. Defensa del patrimonio común: buscan la protección y conservación de la naturaleza (flora, fauna, otros recursos naturales) incitando a pensar que si permanece lo que aún tenemos prevalece la vida humana. 
Desde la Ley colombiana se evidencian grandes esfuerzos por mantener un tipo de desarrollo que demarque un camino sostenible para la naturaleza y el ser humano pero la fuerza del pensamiento sobre crecimiento económico hace que éste se perfile como un imaginario fuertemente instituido, como un imaginario que impide el florecimiento de otros imaginarios instituyentes y opaca aquellas tendencias primitivistas, ecodesarrollistas y a los defensores del patrimonio común.

Aunque puede ser visible, es importante hacer dos anotaciones con respecto a los tres imaginarios catalogados como instituyentes en el párrafo anterior: la primera es que mantienen como centro el ambiente como naturaleza (flora, fauna, otros recursos naturales), y la segunda, que la consideración de instituyentes se le atribuye por ser imaginarios que están en proceso de consolidación y aprehensión por la comunidad colombiana en general, pero, en la medida que estos imaginarios se van materializando y naturalizando (8) en los pensamientos, sentires, acciones de las personas (cuestión que puede estar reflejada en leyes, estatutos, decretos, artículos u otros) estos pasan de ser imaginarios instituyentes a imaginarios instituidos.

Es conveniente aclarar que, para el estudio, fueron tomados como imaginarios instituidos, aquellos que se heredan obligatoriamente de la ley o que estén resguardados por la institución educativa.

Sin embargo, la revisión realizada a los documentos ya mencionados, evidenció que entre estos imaginarios instituidos se perfilan imaginarios instituyentes; por ejemplo, en el grupo de imaginarios instituidos -patrimonio común/ambiental-, la categoría "educación ambiental" encierra diversas alternativas instituyentes. Ambiente como educación, es asumido institucionalmente como la asignatura en los planes de estudio, pero, en los imaginarios instituyentes, las significaciones rompen con estos esquemas convencionales y se orientan hacia la educación ambiental como trasformación constante de las actitudes.

En el grupo de imaginarios instituidos eco-desarrollistas, la categoría "Reconocimiento de la relación entre ser humano y naturaleza" advierte la necesidad de mantener un equilibrio entre el ser humano y la naturaleza, creando nuevas formas de interacción; sin embargo, en los instituyentes, además de mantener la preocupación por las correlacionas expuestas, se amplía esa panorama esa relación "ambiente/naturaleza" a espacios referidos a la convivencia, solidaridad, salud, trabajo y opinión de las organizaciones comunitarias. Su preocupación hacia campos más sociales constituye un movimiento reciente que toma fuerza en medio de los instituidos oficialmente.

En suma, pese a que se mantiene como imaginario central el instituido, en torno a él se construyen nuevas significaciones, las cuales se ubican al margen de lo meramente natural y se entretejen con aspectos como el contexto, lo cultural, el bienestar, el aprendizaje, entre otros. En las nuevas significaciones, ambiente empieza a tener connotaciones de creación y construcción social, empieza a consolidarse como constructo cultural y como posibilidad de ser en el mundo. Es lo que me permite andar "bienestar", es lo que me permite comprender y conocer "aprendizaje", es lo que me permite sentir, imaginar y hacer "contexto".

Este primer acercamiento también evidenció la emergencia de un imaginario radical. El ambiente devenido de la "imaginación" categoría que se ha catalogado como radical, 
en tanto sólo aparece en una ocasión, el escrito manifiesta un pensamiento diferente al resto de los estudiados, rescata la imaginación como un recurso inexplorado e ineludible de la existencia humana, de la cual depende el mundo en toda su extensión, puesto que ella es el recurso humano que renombra, que reconstruye la realidad y que la define. En este sentido, darle tiempo a la imaginación para que construya una nueva sociedad/ambiental, podría ser una sensata alternativa futura.

\section{IMAGINARIOS AMBIENTALES: VOCES EMERGENTES DESDE LA VIDA COTIDIANA}

Este apartado da cuenta del segundo momento de encuentro con los imaginarios ambientales de la Universidad de Caldas, los cuales se dibujan desde entrevistas y encuestas realizadas a la comunidad universitaria. La utilización de estas técnicas permitió centrarse en las manifestaciones íntimas, la sensibilidad, la sociabilidad y los afectos que en el día a día los actores de la cotidianidad universitaria construyen frente al "ambiente". A la vez, ir perfilando paulatinamente los imaginarios sobre ambiente que se construyen en la vida Universitaria. Pese a que se mantienen algunos imaginarios definidos en la teoría formal, en las leyes y normas institucionales ya revisadas (caso de los imaginarios primitivistas, ecodesarrollistas y ambiente como naturaleza), surgen con gran fuerza otros imaginarios como cultura, universo y tecnología.

\section{Ambiente es naturaleza.}

Para la gran masa de la comunidad universitaria, el ambiente se asocia con la naturaleza (flora, fauna, agua, aire, tierra, minerales, etc.). Esta consideración se evidencia en relatos como los siguientes: "Ambiente para mí, es todo lo que tiene que ver con el medio ambiente, el aire que respiras, el agua que tomas, es naturaleza." (9. Estudiante. Ingeniería en Sistemas y Computación.

Semestre)

"Uno siempre relaciona ambiente con la contaminación, con la naturaleza, zonas verdes, la destrucción del medio ambiente". (5. Directora. Departamento. Facultad Ingeniería.)

La fuerza de este imaginario es muy relevante, en tanto un $32 \%$ del total de las respuestas obtenidas en la encuesta están a favor de este concepto. (Ver tabla $\mathrm{N}^{\mathrm{o}} 1$. Ítem 1). De entrada podríamos decir que para este grupo de personas, el ambiente es similar a la naturaleza; hecho que se constituye como normal, como natural y, en tanto ha sido tomada desde el cúmulo de experiencias de estudio, la ley y la vida en general, no es cuestionable ni requiere modificaciones. En consideración a ello, el concebir ambiente como naturaleza está acompañado de otras significaciones como: medio ambiente, contaminación, destrucción de la naturaleza, zonas verdes. Frente a estos acompañantes de ambiente como naturaleza, los entrevistados realizan precisiones en las que medio ambiente adquiere dos connotaciones: la primera es la de considerar que ambiente es como una naranja divida en dos partes iguales, una mitad corresponde a la naturaleza y la otra mitad corresponde al ser humano. La segunda connotación es la de perfilar el "medio ambiente" como los espacios, conductos o canales naturales (flora, fauna, agua, aire, minerales, etc.) necesarios para la vida. La contaminación y la destrucción de la naturaleza vienen a ser 
parte de la mitad de la naranja que corresponde al ser humano y las zonas verdes a la parte de la naranja que le corresponde a la naturaleza. La mitad del ambiente en la que se enmarca al ser humano, (con toda su fuerza destructiva y de contaminación), y la mitad del ambiente en la que se enmarcan los escenarios propicios para la vida, se constituye en la razón para que exista la necesidad de considerar una conciencia ambiental. Las personas que se centran en este grupo, argumentan que la naturaleza existió primero que el ser humano, el ser humano deviene de la naturaleza; por tanto, el ser humano se debe y le debe su existencia a la naturaleza. En este sentido, somos sujetos adaptativos frente a las determinaciones del mundo natural. Maya (1996) (9), considera que el ser humano buscó cobija en la piel de animales, porque las inclemencias y las transformaciones del ambiente lo obligaron. El ser humano actúa sobre la naturaleza porque ella lo exige, pero la forma de actuar sobre ella se ha convertido, desde este imaginario, en una explotación inescrupulosa por parte del ser humano, por tanto hay que separar los dos mundos a fin de hacer que uno sea la razón de ser del otro. La insistencia en esta connotación en términos de que la que mitad debe ser la razón de ser del otro, evidencia la necesidad de que el medio ambiente natural debe ser la razón de ser del medio ambiente humano (conciencia ambiental). Desde este imaginario, se considera que el ser humano es "resultado" de la evolución o transformación de la naturaleza, es un hijo de la madre tierra y, como todo "buen" hijo debe cuidar y proteger a su madre cuando esta está enferma, no causarle la enfermedad. En el sentido anterior, son herederos de este imaginario los movimientos sobre el calentamiento global, las grandes depresiones atmosféricas y tormentas, sequías, indigestiones de gases (huracanes o ciclones), el aumento en volumen y altura de la vejiga de los mares, la contaminación de alimentos, el aire y el agua, el empequeñecimiento de bosques, el resquebrajamiento de la capa de ozono, entre otros; cuestiones que han sido provocadas por los "malos" hijos.

En este sentido, un "buen hijo" gasta menos combustible no renovable, utilizando otros medios para transporte que eviten su consumo, proponiendo energías renovables (plantas eléctricas, generadores eólicos, celdas solares, edificaciones inteligentes y ecológicas), desarrollando procesos de cuidado y protección de la naturaleza (reciclaje, protección de incendios, campañas contra la reducción de gases, contaminación auditiva, entre otros).

En sus significaciones se destacan dos situaciones a considerar: la primera, demarca una gran responsabilidad con la dadora de la vida humana, toda vez que hay que cuidarla para cuidarnos a nosotros como especie, en este mismo sentido surge una segunda consideración, referida al temor ineludible sobre el porvenir de la existencia humana. La férrea idea implícita de "inmortalidad humana" hace que se busquen los responsables de los actos de los "malos hijos" para que sean ellos quienes paguen y no perjudiquen al resto que quiere vivir por más tiempo o reproducir su existencia con su descendencia. En últimas no es por la naturaleza en sí que se debe proteger y conservar, es por el "miedo" de la extinción humana.

El reconocer que el ser humano es hijo de la naturaleza es reconocer que este es "evolución" de ella, pero es una "evolución" estancada, coartada, limitada, ya que controla a toda costa la facultad imaginaria que este posee. Cada vez que el ser humano hace uso de esta facultad provoca "transformaciones" en la naturaleza, transformaciones que en la mayoría de los casos van más en contra que a favor de la naturaleza, según 
este imaginario. Por tanto, es mejor controlar, limitar. Por ello sugerencias como: gastar menos combustible, caminar más o, cuando se innova con algún artefacto en procura de que éste tenga el menor impacto posible sobre la naturaleza, se orientan solamente a proteger la vida humana. Desde este imaginario no se invisibiliza la facultad imaginaria del ser humano, antes se hace visible; desafortunadamente las mayores visibilidades tienen connotaciones negativas (destrucción, contaminación).

En otras palabras, bajo este imaginario no se pide que se aleje o que se deje de usar las creaciones de los "hijos malos", aquellas que destruyen la naturaleza; lo que se pide es que se haga uso en menor proporción y con el menor impacto negativo posible. Esta alternativa es a corto plazo viable, pero a largo plazo, insuficiente. Controlar los impactos, no deja en cero los impactos, sólo retarda la explosión, porque no se pierde continuidad de las acciones.

\section{Ambiente es cultura}

Tanto los documentos institucionales como testimonios y respuestas de las encuestas afloran otra forma de ver el ambiente que se sale de lo netamente natural. Esta cuestión es delimitada como imaginario instituyente y aboga por aspectos culturales. Se centra en aquellas características simbólicas y significantes que identifican a un grupo en particular. Este imaginario es apoyado en un $15 \%$ por la comunidad universitaria (Ver tabla N1. Ítem 1). Frente a este tema se destacan los siguientes trozos de testimonios:

Ambiente "es la relación que se teje con las otras personas. Por ejemplo, el ambiente laboral" (13. Secretaria. Facultad de Ciencias Agropecuarias.)

"Concibo ambiente como un espacio social en el cual uno se desenvuelve, es decir, si nosotros vamos hablar de política entonces el ambiente se torna en leyes, derechos, obligaciones, organización del mundo; si estamos en la universidad entonces el ambiente es académico, entonces fluye el conocimiento, la discusión, la reflexión. Básicamente considero que ambiente sea como eso." (7. Estudiante. Programa Ingeniería en Sistemas y Computación. X Semestre).

Ambiente "es como convivimos en la Universidad, es la relación que se construye con los estudiantes, con los compañeros." (19. Profesora. Facultad Ciencias Agropecuarias) A diferencia del imaginario anterior, aquí desaparece lo natural y se sumerge en el mundo de las relaciones de humanos entre humanos. Las fuerzas de cambio y transformación no son determinadas por la madre tierra, sino por los valores, percepciones, preferencias y comportamientos de los humanos en pro de sí y de su mundo. Este imaginario trae consigo las ideas del contexto del iluminismo, donde cultura y civilización son utilizadas como sinónimos (10) y significan la refinación de las costumbres, lo cual implica, entre otras, el perfeccionamiento progresivo de las leyes, las formas de gobierno, el conocimiento.

Desde este imaginario el ambiente se convierte en aquellos espacios de flujo de emociones, conocimientos, habilidades que construyen el día a día en la Universidad de Caldas. Desde los testimonios de quienes sostienen un imaginario de ambiente como cultura expresan claramente la figura del ser humano como ser que "construye", "reflexiona" y "teje relaciones con las demás personas". El reconocimiento que se le concede al ser humano lo ubica como creador y protagonista de los cambios y 
transformaciones del mundo, es él quién designa las significaciones que le dan existencia.

Algunos análisis llevan a considerar, en primer lugar, que desde este imaginario el ambiente pierde su peso supra-orgánico, al considerarse por encima de los seres humanos, al regir su forma de vida, al ser algo dado inmutable y se trasporta hacia la construcción social (11). Aquí ambiente-cultura es un acto de poiésis humana, por tanto, es susceptible de ser modificado, cambiado, aceptado, rechazado, recreado o radicalmente creado. En esta perspectiva la cultura no le da al ser humano la capacidad de reflexionar, pues el ser humano nace con la facultad de reflexión (12) y por tanto transforma y crea cultura; no es la cultura la que crea el ser, sino el ser humano define su cultura; es esta misma capacidad o facultad de reflexión la que le permite reconocerse como inacabado, cuestionador de sus propias realizaciones y buscador incansable de nuevas significaciones.

En segundo lugar, las expresiones "construcción, reflexión y tejido", se manifiestan como colectivo, invitan a la construcción con el otro, hay reflexión y tejido por la interacción con los demás por la posibilidad de ser en relación, de constituirse en la medida de la constitución del otro; por tanto, son categorías netamente sociales. El hilo invisible que "sujeta", que sirve como vehículo, que sostiene las construcciones, reflexiones o tejidos ambiente-cultura es lo social. Este tejido, en la mayoría de las veces intangible, incuba lo denominado por Castoriadis como colectivo anónimo, aquello que para muchos pareciera que quedara en el aire es el origen del imaginario que se va instituyendo: el ambiente como cultura.

\section{Ambiente es todo lo que está dentro del Universo}

De manera particular, los hallazgos de este segundo acercamiento a los imaginarios sobre ambiente, desde el bullicio de lo cotidiano en la Universidad de Caldas, permitieron consolidar tres grupos de significaciones referidas a ambiente como Universo.

En estas tres categorías del imaginario colectivo, podrían considerarse como radicales/instituyentes, pues hace presencia en las fuentes ya determinadas, pero comienzan a tener peso social. El primer grupo considera el ambiente como una especie de magma, cuyos minerales fundidos son los pensamientos, acciones y sentires de los seres humanos, la tecnología o la naturaleza. En otras palabras, ambiente es todo aquello que está dentro del universo. El 3\% de la población universitaria está de acuerdo con este imaginario, lo cual lo ubica en un buen camino hacia el reconocimiento social (Ver cuadro $\mathrm{N}$ 1. Ítem 1).

"Todo lo que está dentro del universo es ambiente, no importa si es vivo, no vivo, biótico, abiótico, todo es ambiente”. (22. Profesor. Facultad Ciencias Exactas y Naturales)

"Ambiente es todo aquello en lo que estamos circunscritos como seres humanos. La naturaleza, el mismo ser humano y sus creaciones, hasta las influencias cósmicas, todo eso es ambiente" (18. Profesora. Facultad Ciencias para la Salud)

"Ambiente son varias cosas: naturaleza, personas, universo y las relaciones entre todos ellos” (9. Estudiante. Ingeniería en Sistemas Informáticos. VII semestre) 
Como se aprecia, los linderos de la perspectiva ambiental están en todo lo que puede ser considerado universo: la existencia y la relación entre lo vivo y lo no vivo, la naturaleza, el ser humano y sus creaciones, las influencias cósmicas y el universo exterior. Entre los entrevistados que ostentan este imaginario no se hallaron presunciones sobre hijos malos y/o buenos o sobre construcción humana como centro, tal y como se presenta en los imaginarios anteriores. Aquí todo lo que comporta el ambiente/universo mantiene similar rango de importancia: lo tangible y lo intangible, lo vivo y lo no vivo comportan límites inexactos, difusos, incomprensibles para lógicas hegemónicas antes desarrolladas.

Esta perspectiva le impone al ser humano el reto de trasgresión de la explicación científica tradicional y superpone lo no determinado, como escenario de comprensión de los hilos invisibles que constituyen el universo. Bajo este imaginario, el ambiente no es insensible a lo considerado por los entrevistados como contextos (geográficos, políticos, económicos, culturales, naturales, sociales) próximos-locales, nacionales, internacionales, mundiales, universales. Una parcela de realidad siempre mantendrá un inter-juego de construcción y reconstrucción permanente entre los macro y los micros contextos, siempre en relación horizontal con el ser humano. En este sentido, ambiente implica fragmento y holograma del mundo, parcialidad y totalidad, localidad y globalidad.

\section{Ambiente es lo que rodea al ser humano}

El segundo grupo de imaginarios radicales/instituyentes definidos desde el bullicio de lo cotidiano, ubica el ambiente como todo lo que rodea al ser humano. Pese a la similitud con la categoría anterior, las significaciones en este imaginario se orientan a considerar lo que está fuera del ser humano como ambiente: eso que nos influye, nos impregna y en muchas ocasiones nos define. Algunos testimonios al respecto son:

Ambiente es "todo lo que nos rodea, de por sí los inmuebles y las personas que están cerca, los árboles" (11. Estudiante. Enfermería. I Semestre)

Ambiente es "lo que nos rodea, como por ejemplo las personas, la naturaleza, los artefactos...” (10. Estudiante. Agronomía. VIII Semestre)

Ambiente es "el entorno físico que nos rodea...el ambiente construido que es la arquitectura, la tecnología, el medio natural y personas." (20. Profesor. Facultad Artes y Humanidades)

Pese a no ser un imaginario instituido, la categoría "ambiente como universo" es muy fuerte en la comunidad universitaria. Un $38 \%$ asume esta perspectiva. (38\%. Ver tabla $\mathrm{N}^{\mathrm{o}}$ 1. Ítem 1), lo cual lo ubica en un sitial de gran privilegio entre los imaginarios radicales/instituyentes. Radical por emerger en sólo uno de los campos o de las fuentes de información consideradas (el bullicio de lo cotidiano), e instituyente por mantener presencia en la mayoría de la comunidad universitaria.

El ambiente aquí adquiere una connotación de gran poder sobre el ser humano, pues al estar fuera de este, puede ser su referente; el ser humano es asumido con ser pasivo frente a la potencia de lo exterior. Frente a la potencia de la naturaleza fuera del ser 
humano, del universo fuera de éste y de los mismos seres humanos que están fuera de quien es influido.

La "actitud pasiva del sujeto" tal y como lo asume Schaff (1974) establece lo existente como algo ya dado, prefijado y definido, en cuyo escenario el ser humano es totalmente adaptativo. En el sentido anterior, pese a ser un imaginario radical/instituyente, su perspectiva se fundamenta en una vieja herencia del pensamiento occidental respecto de la idea de realidad: "Todos arrastramos de una manera más o menos implícita una forma de concebir la realidad del mundo que nos rodea, y ésta, la mayoría de las veces, es pensada cómo lo que está frente a nosotros, ya dado como tal; a lo que podemos acercarnos, percibir, clasificar, ordenar, interpretar; pero estas acciones no la constituyen sino se acercan a lo dado como cosas y mundo que está ahí independiente de nuestra mirada y acción sobre él’'. (ZAPOLSKY, 2007)

Las cosas desde este imaginario están por fuera del ser humano; pese a ello, no son incomprensibles, pues, se supone que tienen un valor intrínseco, desde el cual se objetiva frente al mundo. El mundo no está hecho de cosas sino de ondas de movimiento que simulan las cosas que nos han enseñado a considerar como reales. Quienes justifican este imaginario, no niegan que sea el ser humano quién da estas designaciones, pero no se consideran de los humanos que designan, que nombran, que determinan, que instituyen, que crean; se consideran de los seres humanos que sufren de la incertidumbre de lo ya definido. Frases como: "Ya todo está inventado", "tenemos que estar alerta para lo que se presente", son comunes en este grupo. Cuando utilizan la frase "ya todo está inventado", no se refieren explícitamente a que el ser humano ya no puede crear, o en este caso inventar, sino a que ellos no ven necesario incursionarse en las tareas que acarrean actividades creativas. Cuando les fluye alguna idea, de inmediato la asocian con una ya dicha; por tanto se niegan su propia imaginación y niegan la singularidad creativa de ser humano.

\section{Ambiente es tecnología}

El 13\% de la comunidad apoya este imaginario radical/instituyente (Ver tabla $\mathrm{N}^{\mathrm{o}} 1$. Ítem 1). Los testimonios registrados por los investigadores manifiestan que los entrevistados quisieran un mundo definido por las "supuestas" comodidades de los desarrollos tecnológicos orientadas a facilitar muchos procesos y tareas del ser humano. En sus perspectivas se dibuja, por ejemplo, una Universidad dotada con salas de sistemas que faciliten la comunicación, las redes y las tareas de la academia y la administración de la Universidad de Caldas.

"Ambiente es lo que nos rodea y va ligado al bienestar, o sea, facilidades de comunicación como los computadores, el internet, el teléfono, comodidades como una oficina bien dotada con escritorio, sillas, marcadores, tableros, impresora, etc." (3. Director. Programa. Facultad Artes y Humanidades)

"Elementos materiales: salones, computadores, tableros electrónicos, software educativos, laboratorios con mucha tecnología, todo esto es ambiente para mi"(23. Estudiante. Ingeniería de Alimentos. VI Semestre) 
Ambiente es "el estado donde uno se desenvuelve, si la oficina está limpia, si trabajo con los elementos necesarios...lapiceros, calculadora, cosedora, carpetas para archivar, internet, e-mail, etc." (15. Funcionario. Oficios Bibliotecarios. Facultad Ciencias Jurídicas y Sociales)

Este imaginario se mueve bajo dos aspectos: el primero hace alusión al estado tecnológico de los espacios de encuentro social y, el segundo, a la transición del ambiente como naturaleza (flora, fauna, minerales) a un ambiente virtual. Con relación al primer aspecto, los entrevistados manifiestan mediante expresiones como: "Ahora nos podemos ver por las mini cámaras de los computadores y al tiempo hablar, como las tele-conferencias"... "Así, estemos a kilómetros de distancia nos podemos ver y escribir, y en algunos casos hablar paralelamente", que se está configurando un nuevo espacio de encuentro social - el cyberespacio-, en el cual, el cara a cara exige más que la presencia física. De hecho, la presencia física está presente, pero de forma diferente; en ella los cuerpos se ven y comunican, y aunque las percepciones de algunos sentidos como el olfato o la sensación del tacto no están presentes, muchos programas cibernéticos ya están aproximando esta posibilidad desde otras realidades que, aunque virtuales, pueden ser percibidas "como si fueran reales".

En este caso, la exigencia está más orientada hacia la retracción y la aprehensión de las realidades dadas en los encuentros sociales mediados por algún campo tecnológico. Son muchas las preguntas que surgen ante esta forma de concebir las realidades: ¿Qué tanto se altera la realidad cuando se dispone sólo de dos sentidos- auditivo y visual- para captarla?, ¿qué sentidos se necesitan para captar la realidad? Es evidente, en este imaginario, el poder de la imaginación y el surgimiento de nuevas formas simbólicas que fungen como acuerdos sociales para asumirlas como realidad. Una realidad virtual, pero al fin y al cabo realidad.

A decir de quienes asumen esta posibilidad, los nuevos espacios de encuentro social generan lazos fuertes entre quienes se encuentran, pues la aprehensión de la realidad no está limitada a los sentidos convencionales sino que en ellos existe un enorme esfuerzo de imaginación y fantasía. No obstante queda para juicios futuros los entendimientos al respecto.

En relación con el segundo aspecto, se halló que ambiente como tecnología proyecta una nueva naturaleza, no invisibilización de ella, sino construcción de nuevos espacios naturales; se mueve bajo la idea de transferencia o paso de naturaleza viva a una naturaleza artificial.

"En un futuro próximo no van a existir las plantas como ahora las vemos, van a ser plásticas u hologramas” (8. Estudiante. Filosofía y Letras. IV semestre)

Un futuro proyectado desde lo artificial y lo virtual. Un mundo quizá abierto a otros escenarios aún inexplorados de la vida humana, donde las sensaciones no sean la base del reconocimiento de la realidad, sino que ellas están definidas por la imaginación, el transporte de nuestras sensaciones a lo virtual/artificial.

El mundo natural equivale, en esta categoría imaginaria, a lo virtual y artificial. En realidad, lo virtual y/o artificial no corresponde al mundo de lo irreal, sino que es otra forma de realidad. De hecho las propuestas de mundos virtuales tienen ya importantes 
desarrollos en los juegos de redes. En ellos, se crean mundos virtuales que pueden ser reconstruidos permanentemente de acuerdo a los intereses "siempre guiados" de los jugadores. El Erepublik, por ejemplo, creado por Alexis Bonte, es un universo mitad juego y mitad red social, en el cual el jugador crea los universos imaginarios más insólitos (13).

En el marco de los mundos artificiales existe un ejemplo muy fehaciente respecto de la forma como se busca construir mundos inteligentes que reemplacen incluso la creación del ser humano. Son muy reconocidos en este ámbito trabajos de inteligencia artificial como el de Harold Cohen con AARON, un computador dibujante (14).

Hay que reconocer que desde los tiempos de Platón y Aristóteles ya se contemplaba el mundo artificial, aunque con connotaciones especificas. Mientras para Platón lo artificial eran imitaciones de algo natural, de algo genuino u original, que no alcanzaban el valor de real, para Aristóteles no representaba imitaciones de algo dado previamente, sino que eran autenticas invenciones, representaban algo nuevo, no una simple e imperfecta copia de un prototipo.

El imaginario sobre ambiente como tecnología inspira la idea ensoñadora de un mundo computarizado. Los entrevistados que fundamentan este imaginario son constructores del imaginario que hace época - sociedad contemporánea-. La cual explicita como necesidad y como oportunidad de desarrollo el uso y la creación de nuevas tecnologías.

\section{ALGUNOS LINEAMIENTOS PARA EL ABORDAJE AMBIENTAL EN LA UNIVERSIDAD}

Desde los hallazgos se evidencian algunas posibilidades que son importantes para tener en cuenta a la hora de definir programas o políticas ambientales en la Universidad de Caldas.

- Cuando se analiza la Ley colombiana referente al ambiente, se muestra una perspectiva eco-desarrollista donde se intenta articular la perspectiva de un desarrollo ambiental primitivista con un desarrollo cuya filosofía es la acumulación de capital. Pese a ello, en las formas de operacionalizar o de poner en escena este imaginario instituido, se sigue hablando del imaginario como protección y conservación de la naturaleza.

- En el Proyecto Educativo Institucional se muestra cómo los imaginarios vislumbrados desde la normatividad han sido acogidos, trasformados y filtrados por la comunidad universitaria; esto se evidencia en: 1. La aprehensión en algunos planes de estudio, donde se ubican aspectos referidos la educación ambiental, 2. En los pensamientos plasmados en la Revista Institucional Luna Azul y 3. En algunas investigaciones realizadas por profesores y estudiantes de la Universidad de Caldas (15) .

No obstante este seguimiento, la comunidad explora otras categorías imaginarias: ambiente como aspecto de la naturaleza a explorar, como contexto cultural, como bienestar, como educación ambiental y como ambiente de aprendizaje. 
- Existe una estrecha relación entre los imaginarios contenidos en el PEI y los imaginarios plasmados en la producción académica revisada desde los artículos de la Revista Institucional Luna Azul; sin embargo, estos imaginarios manifiestan una permanente movilidad hacia nuevas perspectivas imaginarias como la categoría "educación ambiental" la cual mantiene la esencia intencional, pero su forma de materializarse va generando instituyentes diversos en el mundo educativo.

- Haciendo un análisis de la temporalidad de las categorías imaginarias extraídas desde la Revista se encuentran fuertemente instituidas: Educación Ambiental, inventarios bibliográficos y reconocimiento de la relación entre el ser humano y la naturaleza, las que han hecho presencia entre 8 y 9 años. Otras, con una relativa importancia, han permanecido de forma intermitente en los escritos entre cuatro y cinco años, como el caso de ambiente como arquitectura, naturaleza y ser humano, protección natural, legislación y desarrollo sostenible. Por último, encontramos categorías cuya presencia es menor de tres años. Aunque para algunos esto puede ser muestra de debilidad en sus pensamientos, para otros es signo de imaginarios radicales, es señal de pensamientos nuevos y por tanto desarrollados por pocos. En esta categoría se resalta un imaginario que deja a la facultad poiésica del ser humano la posibilidad de articular todos los escenarios posibles donde tiene cabida la experiencia ambiental.

Este aspecto es importante pues muestra que los imaginarios son extremadamente móviles y que pueden ser construidos y reconstruidos, lo cual es una ventaja relativa en términos de la posibilidad educativa de las relaciones ambientales.

- En cuanto a los estudios científicos realizados en la Universidad de Caldas respecto al ambiente, se develaron dos categorías imaginarias: la primera referida a la identificación de problemas ambientales, sus responsables y la protección de la naturaleza. Esta categoría acoge los parámetros mencionados en el PEI y, por ende, las normatividades ambientales. Una segunda categoría reconoce aspectos como: pensamiento ambiental, ambiente y aprendizaje y, mujer Vs desarrollo sostenible, lo cual concuerda con las adecuaciones y modificaciones que la comunidad universitaria ha adoptado sobre las perspectivas de ambiente contenidas en el PEI. Las intenciones de estas significaciones imaginarias develan un nuevo sentido al estudio sobre ambiente: ambiente no sólo es naturaleza y protección por parte del ser humano, es una cuestión de pensamiento, de aprendizaje y de género.

Aquí las pretensiones rompen la unidireccionalidad y se encausan en un movimiento instituyente, es decir, sus horizontes plantean aspectos poco explorados y con pocos seguidores, pero engendran nuevas posibilidades de estudio que a medida que se popularicen o se socialicen pueden llegar a ser instituidos.

- Cuando se examina la realidad ambiental desde la vida cotidiana se destacan cinco categorías imaginarias: 1. Ambiente como naturaleza 2. ambiente como entorno 3. Ambiente como cultura 4. Ambiente como tecnología y 5. Ambiente como universo. 
El ambiente como naturaleza es común tanto en las leyes del Estado como en el Proyecto Educativo Institucional y tiene una fuerza en la práctica social del $32 \%$, lo cual lo ubica como una categoría de gran relevancia. El ambiente como entorno, pese a que no tiene una fuerza instituida en la ley y el PEI, posee gran potencia en la práctica social con un $38 \%$, por lo cual se considera como un imaginario altamente instituyente en proyección a ser considerado imaginario instituido. Si bien estas categorías imaginarias tienen una gran fuerza en la práctica social, ello no significa que las políticas o programas a realizar en la universidad deban estar orientadas a favorecer sólo estos imaginarios; por el contrario, las teorías sobre imaginarios asumen que es importante tener en cuenta las emergencias, o sea, los imaginarios radicales, para lograr la movilidad que las sociedades e instituciones actuales requieren. (Ver: Shoter 1993).

El estudio de la vida cotidiana muestra que ambiente como cultura, tecnología y universo son, a propósito, imaginarios radicales, que comienzan a emergen en las prácticas cotidianas universitarias. Es importante considerar que en las producciones escritas e investigaciones analizadas mostraron también imaginarios radicales como educación ambiental, facultad autopoiesica, bienestar, ambientes de aprendizaje.

Algunos aspectos estadísticos muestran la forma como la comunidad universitaria desarrolla sus acercamientos al concepto de ambiente:

- El $76 \%$ de la comunidad educativa accede al conocimiento del ambiente, fundamentalmente, a través de la institución educativa, considerando la familia como un agente de gran importancia; entre tanto, sólo el $24 \%$ asume que aprende autónomamente. (Ver tabla $\mathrm{N}^{\mathrm{o}}$ 1. Ítem. 2)

- El 73\% dice no conocer espacios de reflexión sobre ambiente propiciados por la Universidad (Ver tabla $\mathrm{N}^{\circ}$ 1. Ítem. 4). El 77\% dice no conocer documentos elaborados por la Universidad de Caldas acerca de ambiente. (Ver tabla $\mathrm{N}^{0} 1$. Ítem. 5).

- La mayoría de las personas encuestadas (90\%) consideran que la mejor forma de aprender sobre ambiente sería a través de talleres vivenciales y procesos de Investigación, Acción, Participación -I.A.P- (Ver tabla N ${ }^{0}$ 1. Ítem. 3).

\section{APERTURAS (16)}

\section{Reflexión ambiental}

Se trata del reconocimiento de la amplitud de miradas y la complejidad de pensamiento que se compactan en la vida ambiental universitaria. Por tanto la Universidad debe contribuir en un verdadero alfabetismo de la reflexión ambiental, construyendo espacios críticos frente al pensamiento único y la desnaturalización de la realidad -imaginarios instituidos-.

\section{Contrato ambiental}

Se trata de la inclusión o de la participación activa de la comunidad universitaria, tanto en la definición como en el establecimiento de responsabilidades y compromisos frente a la vida ambiental universitaria. Por tanto la Universidad debe generar espacios de 
participación masiva a fin de forjar compromiso y sentimiento de pertenencia frente a la vida ambiental de la Universidad.

\section{Proyecto ambiental}

Se trata de vincular entre sí el mundo académico, el mundo de la vida y el mundo ambiental, bajo la idea de recuperar y potenciar la capacidad innovadora. Por tanto la Universidad debe co-fundar, promover la construcción de un proyecto ambiental propio que permita dibujar una vida ambiental universitaria -futura- que no dependa de lo instituido.

\section{TÉCNICAS}

- Talleres

- Investigación Participativa

- Desarrollo Tecnológico

- Análisis integrado

- Sistema de información

\section{ESTRATEGIAS}

- Fortalecimiento de procesos participativos

- Fomento de la identificación, conservación y difusión de tipologías arquitectónicas que expresen las particularidades ambientales de la Universidad.

- Promoción de mecanismos de concertación.

- Desarrollo de investigación ambiental institucional con connotación de participativa.

- Ordenamiento territorial y administrativo de manera ambiental.

- Promoción de la creación de zonas de reserva natural universitaria.

- Creación de sistema de información ambiental.

\section{CAPACIDADES A DESARROLLAR}

- Valoración de la diversidad integrada. (Cultural, natural, arquitectónica, etc.)

- Búsqueda permanente de nuevas realidades ambientales.

- Comprensión de la interrelación entre vida cotidiana, vida ambiental y vida universitaria.

\section{BIBLIOGRAFÍA}

- ARISTÓTELES. (1970). Physics. Clarendon. (Traducción: W. Charlton).

- BLUM de B, C. (1993) Última oportunidad. Ministerio del Medio Ambiente. Cali. Colombia.

- BRETON, A. (1990). Manifestes du surréalisme. Ed. France Loisirs. Francia. BRIONES, A, G. (1994). Incompatibilidad de paradigmas y compatibilidad de técnicas en ciencias sociales. En: Revista de Sociología 9, Departamento de Sociología de la Facultad de Ciencias Sociales, Universidad de Chile, Santiago, 
1994,

pp.

25-33.

CARRIZOSA, U.J. (1992) La política ambiental en Colombia. Desarrollo sostenible y democratización. Bogotá - Colombia, FESCOL y CEREC.

- CASSIRER, E. (1971) Filosofía de las formas simbólicas. Tomo 1. México. DF: Fondo de cultura económica.

- CASTORIADIS, C. (1997). Ontología de la creación. Bogotá. Colombia. Servigraphic.

laberinto. Barcelona. España. Gedisa.

- _ (1983).La institución imaginaria de la sociedad, 1.Marxismo y teoría revolucionaria, 2. El imaginario social y la institución, Barcelona, Tusquets, 1983, 1989 [Traducción de Abtoni Vicens, vol. 1].

- . (1989).La institución imaginaria de la sociedad, 1. Marxismo y teoría revolucionaria, 2. El imaginario social y la institución, Barcelona, Tusquets, 1983, 1989 [Traducción de Marco-Aurelio Galmarini, vol.2].

Nueva Visión. . (1992) Psicoanálisis, proyecto y elucidación, Buenos Aires, . (1994) El destino de los totalitarismos. En: Los dominios del hombre: Las encrucijadas del laberinto. Barcelona. España. Gedisa.

- COLOMBIA. Presidencia de la República. Constitución Política de Colombia. Bogotá. Imprenta Nacional, 1991.

- DECLARACIÓN DE UNESCO. (1982). Declaración de México sobre las políticas culturales. Conferencia mundial sobre las políticas culturales. México. D. F.

- DECRETO No 1743 de agosto de 1994.

- DIJSKTERHUIS, C. (1986). The Mechanization of the World Picture. Princeton.

- DURAND, G (1968) La imaginación simbólica. Buenos Aires, Argentina. Amorrortu.

- EINSTEIN, A. (2000). Mis creencias. Elaleph.

- ESCOBAR, J.C. (2000). Lo imaginario entre las ciencias sociales y a historia. Medellín. Colombia, EAFIT.

- FEHÉR, M. (1998). Lo natural y lo artificial (un ensayo de clarificación conceptual). En: Revista internacional de filosofía. Vol. XVII. No 3.

- GIORDANO, B. (1997). Mundo, Magia, Memoria. Madrid. España. Biblioteca Nueva.

- GOFFMAN, E. (1993) La presentación de la persona en la vida cotidiana. Buenos Aires. Argentina. Amorrorrtu.

- HELlER, A. (1972) Historia y vida cotidiana: Aportación a la sociología socialista. México. Traducción de Manuel Sacristán. Grijalbo

- LEY 115 de 1994. Bogotá.

- KROEBER, A. (1995). Lo supraorgánico. En: Kahn, J.S. (Comp). El concepto de cultura. Barcelona. Anagrama

- MARQUEZ, G. (1998) Un enfoque de sistemas sobre la sostenibilidad. En: La gallina de los huevos de oro. Debate sobre el concepto de desarrollo sostenible. Ecofondo-Cerec. Santafé de Bogotá. Colombia.

- MAYA, A. A. (1996) La fragilidad ambiental de la cultura. Universidad Nacional. Bogotá. Colombia. 
- MURCIA, P, N. (2006). Vida Universitaria. Un estudio desde los imaginarios de profesores y estudiantes. Manizales. CINDE, Universidad de Manizales. Tesis doctoral.

- MURCIA, P. N \& JARAMILlO, E. L.G. (2007) Complementariedad etnográfica. Una posibilidad para el diseño de investigación cualitativa. Segunda edición. Kinesis. Armenia. Colombia.

- NOGUERA, A. P. (1998) Escisión y reconciliación. Universidad Nacional de Colombia. Manizales. Colombia

- OSPINA, O. L. E \& MURCIA, P. N. (2007). Imaginarios ambientales que irradian la Universidad de Caldas. En: Revista electrónica Luna Azul, No 25. Dirección Electrónica: http://lunazul.ucaldas.edu.co/

- POLANYI, K. (1944). The great transformation. [Traducción española: La gran transformación. 1989. Ed. La piqueta. Madrid].

- PROYECTO EDUCATIVO INSTITUCIONAL DE LA UNIVERSIDAD DE CALDAS. Manizales. Imprenta Universidad de Caldas, 1996 - 2010.

- RAMIREZ, V. M. (1998) El concepto de desarrollo humano sostenible. En: La gallina de los huevos de oro. Debate sobre el concepto de desarrollo sostenible. Ecofondo - Cerec. Santafé de Bogotá - Colombia.

- SCHAFF, A. (1974) Historia y verdad. México. D. F. Grijalbo

- SAHLING, M. (1990). Uso y abuso de la biología: Crítica antropológica de la sociobiología. Siglo XXI. Madrid.

- SHERPARD, P. (1998). Comino home to the pleistocene. Ed. By Florence. Washinton. D. C. ISLAND PRESS/Shearwater Books.

- SHOTTER, J. (1993). Realidades conversacionales. La construcción de la vida a través del lenguaje. Ed. Amorrortu. Buenos Aires.

- THOMPSON, J. B. (2002). [1990]. Ideología y cultura moderna.Teoría crítica social en la era de la comunicación de masas. Ed. División de Ciencias Sociales y Humanidades de la Universidad Autónoma Metropolitana. Unidad Xochimilco. México.

- ZAPOLSKY, L. El concepto de realidad en la obra de C. Castoriadis. http://www.magma-net.com.ar/conceptorealidad.htm (28-05-2007)

- GOFFMAN, E. (1993) La presentación de la persona en la vida cotidiana. Ed. Amorrorrtu. Buenos Aires.

\section{NOTAS}

1. Este primer acercamiento se compartió con la comunidad académica en el articulo "Imaginarios ambientales que irradian la Universidad de caldas" de Ospina y Murcía (2007), publicado en la revista electrónica Luna Azul Número 25. http://lunazul.ucaldas.edu.co/index.php?option $=$ com frontpage\&Itemid $=1$

2. Imaginario instituido: esquemas de inteligibilidad que permanecen y orientan un grupo de personas. Ver Cornelius Castoriadis La institución imaginaria de la sociedad. Vol. 1 (1983) y Vol. 2 (1989).

3. Imaginario instituyente: Tendencias sentidas, que están en proceso de construcción colectiva. Ver Cornelius Castoriadis La institución imaginaria de la sociedad. Vol. 1 (1983) y Vol. 2 (1989). 
4. Imaginario radical: Significaciones presentes en personas particularesPsiquis/soma en ebullición. Ver Cornelius Castoriadis La institución imaginaria de la sociedad. Vol. 1 (1983) y Vol. 2 (1989).

5. La información de la población correspondiente a los profesores fue suministrada por la Oficina de Desarrollo Docente, la de los estudiantes por Registro Académico y la de los otros funcionarios por la Oficina de Desarrollo Humano de la Universidad de Caldas durante el 2006.

6. Cada muestra fue ponderada según carrera para los estudiantes; tipo de vínculo laboral para los profesores (planta $u$ ocasional) $y$ otros funcionarios (trabajadores, vigilantes, técnicos, secretarias, etc.)

7. Para ampliar sobre el tema ver autores como: Kart Polanvi (1944), Marshall Sahlins (1990), Paul Shepard (1998).

8. Se habitúan de manera tal que no son cuestionados, por tanto no son transformados por largos períodos de tiempo.

9. MAYA, A, A. (1996). La fragilidad ambiental de la cultura. Ed. Universidad Nacional de Colombia. Bogotá - Colombia.

10. Para ampliar sobre cultura y civilización en el contexto del Iluminismo leer a Thompson, J. B. (2002). [1990]: Ideología y cultura moderna. Teoría crítica social en la era de la comunicación de masas. División de Ciencias Sociales y Humanidades de la Universidad Autónoma Metropolitana - Unidad Xochimilco. México. Pág. 186

11. Autor que justifica la posición de la cultura como supraorgánica: Kroeber, A. (1995). Lo supraorgánico. En: Kahn, J.S. (Comp). El concepto de cultura. Anagrama Barcelona.

12. Posición contraria a la declaración realizada por la UNESCO en 1982, México: la cultura da al hombre la capacidad de reflexionar sobre sí mismo. Es ella la que hace de nosotros seres específicamente humanos, racionales, críticos y éticamente comprometidos. A través de ella discernimos los valores y efectuamos opciones. A través de ella el hombre se expresa, toma conciencia de sí mismo, se reconoce como un proyecto inacabado, pone en cuestión sus propias realizaciones, busca incansablemente nuevas significaciones, y crea obras que lo trascienden. (UNESCO, 1982: Declaración de México)

13. Ver: http://www.elmundo.es/navegante/2008/05/30/juegos/1212173775.html

14. Ver: http://www.kurzweilcyberart.com/

15. Ver Ospina y Murcia (2007).

16. Estas aperturas, técnicas, estrategias y capacidades a desarrollar, son consideradas desde la potencia de las respuestas tomadas, tanto de las entrevistas como de las encuestas, realizadas a la comunidad universitaria.

\section{ANEXOS}

\section{Fuentes de información institucional}

- Revistas Luna Azul 1995 - 2006

- Catálogos de Investigación 1996 - 2006

- Proyecto Educativo Institucional 1996 - 2010

- Bases de datos de la Vicerrectoría de Investigaciones y Postgrados. U de C 
- Informes finales de Investigaciones. Archivos. U de C.

- Comunidad Universitaria. U de C.

Tabla 1. Resumen de resultados estadísticos.

\begin{tabular}{|c|c|c|c|c|c|c|}
\hline \multirow{3}{*}{\begin{tabular}{|c|} 
\\
ITEM \\
Ítem 1.
\end{tabular}} & \multirow{3}{*}{$\begin{array}{c}\text { RESPUESTA } \\
\text { Naturaleza }\end{array}$} & \multicolumn{3}{|c|}{ POBLACION } & \multirow{2}{*}{\multicolumn{2}{|c|}{ TOTALES }} \\
\hline & & \multirow{2}{*}{$\frac{\text { Estudiantes }}{171}$} & \multirow{2}{*}{$\mid$\begin{tabular}{|c|} 
Profesores \\
97
\end{tabular}} & \multirow{2}{*}{\begin{tabular}{|c|}
$\begin{array}{c}\text { Personal } \\
\text { administrativo } \\
\text { y de servicios } \\
\text { generales }\end{array}$ \\
50 \\
\end{tabular}} & & \\
\hline & & & & & 318 & $32 \%$ \\
\hline \multirow{5}{*}{ ¿Qué es ambiente? } & Tecnología & 71 & 39 & 24 & 134 & $13 \%$ \\
\hline & Cultura & 74 & 51 & 21 & 146 & $14 \%$ \\
\hline & Entorno & 238 & 103 & 38 & 379 & $38 \%$ \\
\hline & Universo & 14 & 14 & 4 & 32 & $3 \%$ \\
\hline & Totales & 568 & 304 & 137 & 1009 & $100 \%$ \\
\hline \multirow{4}{*}{$\begin{array}{c}\text { ¿Cómo fue } \\
\text { conociendo sobre } \\
\text { ambiente? }\end{array}$} & $\begin{array}{l}\text { Institución } \\
\text { educativa }\end{array}$ & 371 & 194 & 70 & 635 & $63 \%$ \\
\hline & Familia & 70 & 40 & 23 & 133 & $13 \%$ \\
\hline & Autónomamente & 127 & 70 & 44 & 241 & $24 \%$ \\
\hline & Totales & 568 & 304 & 137 & 1009 & $100 \%$ \\
\hline \multirow{10}{*}{$\begin{array}{c}\text { Ítem } 3 . \\
\text { ¿Cómo cree usted } \\
\text { que puede } \\
\text { aprender sobre } \\
\text { ambiente dentro de } \\
\text { la Universidad? }\end{array}$} & $\begin{array}{c}\text { Talleres } \\
\text { vivenciales }\end{array}$ & 267 & 96 & 66 & 429 & $43 \%$ \\
\hline & $\begin{array}{l}\text { Procesos de } \\
\text { Investigación, } \\
\text { acción, } \\
\text { participación. }\end{array}$ & 249 & 169 & 57 & 475 & $47 \%$ \\
\hline & $\begin{array}{c}\text { Textos } \\
\text { tradicionales. } \mathrm{Ej} \text {. } \\
\text { Cartillas, } \\
\text { boletines. }\end{array}$ & 23 & 16 & 11 & 50 & $5 \%$ \\
\hline & $\begin{array}{c}\text { Mediante alguna } \\
\text { herramienta } \\
\text { multimedia }\end{array}$ & 29 & 23 & 3 & 55 & $5 \%$ \\
\hline & Totales & 568 & 304 & 137 & 1009 & $100 \%$ \\
\hline & $\begin{array}{c}\text { Talleres } \\
\text { vivenciales }\end{array}$ & 267 & 96 & 66 & 429 & $43 \%$ \\
\hline & $\begin{array}{l}\text { Procesos de } \\
\text { Investigación, } \\
\text { acción, } \\
\text { participación. }\end{array}$ & 249 & 169 & 57 & 475 & $47 \%$ \\
\hline & $\begin{array}{c}\text { Textos } \\
\text { tradicionales. Ej. } \\
\text { Cartillas, } \\
\text { boletines. }\end{array}$ & 23 & 16 & 11 & 50 & $5 \%$ \\
\hline & $\begin{array}{c}\text { Mediante alguna } \\
\text { herramienta } \\
\text { multimedia }\end{array}$ & 29 & 23 & 3 & 55 & $5 \%$ \\
\hline & Totales & 568 & 304 & 137 & 1009 & $100 \%$ \\
\hline
\end{tabular}




\begin{tabular}{|c|c|c|c|c|c|c|}
\hline \multirow{3}{*}{$\begin{array}{c}\text { Ítem } 4 . \\
\text { ¿Conoce espacios } \\
\text { de reflexión sobre } \\
\text { ambiente } \\
\text { propiciados por la } \\
\text { Universidad de } \\
\text { Caldas? }\end{array}$} & SI & 147 & 94 & 33 & 274 & $27 \%$ \\
\hline & NO & 421 & 210 & 104 & 735 & $73 \%$ \\
\hline & Totales & 568 & 304 & 137 & 1009 & $100 \%$ \\
\hline \multirow{5}{*}{$\begin{array}{c}\text { Ítem 5. } \\
\text { ¿Cuántos } \\
\text { documentos } \\
\text { elaborados por la } \\
\text { Universidad de } \\
\text { Caldas acerca de } \\
\text { ambiente conoce? } \\
\text { (revistas, } \\
\text { investigaciones, } \\
\text { políticas, PEI, } \\
\text { resoluciones, etc.) }\end{array}$} & 1 y 2 documentos & 57 & 68 & 27 & 152 & $15 \%$ \\
\hline & 3 documentos & 5 & 13 & 4 & 22 & $2 \%$ \\
\hline & $\begin{array}{c}\text { Más de } 4 \\
\text { documentos }\end{array}$ & 18 & 38 & 7 & 63 & $6 \%$ \\
\hline & No conoce & 488 & 185 & 99 & 772 & $77 \%$ \\
\hline & Totales & 568 & 304 & 137 & 1009 & $100 \%$ \\
\hline
\end{tabular}

